\title{
Lidil
}

Revue de linguistique et de didactique des langues

$54 \mid 2016$

La phrase en production d'écrits, approches nouvelles en didactique

\section{La conception de la phrase chez les enseignants et les élèves québécois du primaire}

The Notion of Sentence for Quebec Elementary School Teachers and Students

Pascale Lefrançois, Isabelle Montésinos-Gelet et Dominic Anctil

\section{(2) OpenEdition}

\section{Journals}

Édition électronique

URL : http://journals.openedition.org/lidil/4056

DOI : $10.4000 /$ lidil.4056

ISSN : 1960-6052

Éditeur

UGA Éditions/Université Grenoble Alpes

Édition imprimée

Date de publication : 20 novembre 2016

Pagination : 75-91

ISBN : 978-2-84310-339-1

ISSN : $1146-6480$

Référence électronique

Pascale Lefrançois, Isabelle Montésinos-Gelet et Dominic Anctil, «La conception de la phrase chez les enseignants et les élèves québécois du primaire », Lidil [En ligne], 54 | 2016, mis en ligne le 01 janvier 2017, consulté le 29 octobre 2020. URL : http://journals.openedition.org/lidil/4056 ; DOI : https:// doi.org/10.4000/lidil.4056 


\title{
La conception de la phrase chez les enseignants et les élèves québécois du primaire
}

\author{
Pascale Lefrançois, Isabelle Montésinos-Gelet \\ et Dominic Anctil*
}

\begin{abstract}
RÉSUMÉ
Bien que la notion de phrase soit centrale dans la grammaire française et dans les programmes d'enseignement, peu de données sont disponibles pour illustrer la conception qu'en ont les élèves du primaire et leurs enseignants. Cet article rapporte les résultats d'une recherche collaborative réalisée en contexte québécois, qui a permis de décrire la performance d'élèves de la $1^{\text {re }}$ à la $6^{\mathrm{e}}$ année du primaire ainsi que celle de leurs enseignants à une entrevue sur la notion de phrase, les arguments utilisés par les uns et les autres pour justifier leurs jugements et le lien entre les savoirs des élèves et ceux des enseignants à travers une étude de cas réalisée dans une classe.
\end{abstract}

\begin{abstract}
Though the notion of sentence is crucial in French grammar and in the curriculum, there is a lack of data on how elementary school students and teachers conceive this notion. This article presents the results of an action-research carried on in Quebec. This research allowed to describe grade-one-to-six students' and their teachers' performance at an interview on the notion of sentence, arguments they used to justify their grammaticality judgments, and relationships between students' and teachers' knowledge through a case study.
\end{abstract}

\section{Introduction}

La compétence à écrire inclut, notamment, une importante composante linguistique (groupe DIEPE, 1995), qui fait elle-même appel à plusieurs dimensions : l'orthographe lexicale et grammaticale, la syn-

* Université de Montréal. 
taxe et la ponctuation, le lexique et la pragmatique (Riegel, Pellat \& Rioul, 2004). La description de la langue enseignée actuellement dans les écoles québécoises, appelée «nouvelle grammaire» ou «grammaire moderne », accorde une place plus importante qu'auparavant aux dimensions syntaxiques, sous l'influence des recherches en linguistique. En effet, l'étude du fonctionnement de la phrase est au centre des théories linguistiques des années 1950, dont la transposition didactique teinte les programmes québécois de français depuis 1995 (Desnoyers, 2001). Selon Nadeau et Fisher (2006), trois des grammaires scolaires destinées au secondaire parmi les plus utilisées au Québec consacrent entre 45 et $69 \%$ de leur contenu à la syntaxe; cette proportion est moins élevée dans les manuels destinés au primaire, vu l'âge des élèves et le contenu du Programme de formation de l'école québécoise. Toutefois, la Progression des apprentissages au primaire en français, langue d'enseignement (MELS, 2009), qui précise les contenus linguistiques du programme, consacre à la syntaxe $26 \%$ de ses pages sur la compétence à écrire, ce qui en fait la section la plus importante parmi les six que comporte le document.

Malgré cette importance accrue de la syntaxe dans les programmes et le matériel didactique, peu de données sont disponibles pour décrire les connaissances en syntaxe des élèves, plus particulièrement au primaire. Une des rares recherches récentes à s'être penchées sur la maitrise de la langue des élèves québécois de fin de $6^{\mathrm{e}}$ année du primaire (Lefrançois, Laurier, Lazure \& Claing, 2008) a montré 1) que la syntaxe est la section la moins bien réussie dans un questionnaire à choix multiple évaluant les connaissances en orthographe lexicale, en orthographe grammaticale, en syntaxe et en lexique et 2) que les erreurs de syntaxe et de ponctuation constituent $30 \%$ des erreurs en production écrite. De même, les dernières données disponibles sur la performance des élèves de $6^{\mathrm{e}}$ année à l'épreuve obligatoire d'écriture du Ministère révèlent que, des cinq critères d'évaluation des productions écrites, celui portant sur la syntaxe et la ponctuation est le moins bien réussi (MELS, 2012). Ainsi, en dépit de l'importance que lui accordent les nouveaux programmes, la syntaxe apparait comme un aspect de la langue qui pose problème aux élèves du primaire, notamment en écriture.

Au cœur de la syntaxe se situe la notion de phrase. Or, dans les descriptions grammaticales actuelles, il existe plusieurs «phrases», qui décrivent des réalités distinctes, souvent imbriquées les unes dans les autres (Boivin \& Pinsonneault, 2008; Riegel et coll., 2004). Mentionnons, par exemple, la phrase syntaxique $(\mathrm{P})$, qui est définie en termes de constituants (sujet, prédicat et, facultativement, complément(s) de 
phrase); la phrase syntaxique subordonnée, P qui dépend d'une autre et y joue presque toujours une fonction grammaticale; la phrase syntaxique autonome, P qui ne dépend d'aucune autre et n'a pas de fonction grammaticale; la phrase de base, modèle théorique permettant de décrire la structure de presque toutes les phrases réalisées, dont les constituants sont placés dans l'ordre canonique (sujet, verbe, complément(s) du verbe, attribut, complément(s) de phrase); enfin, sur un tout autre plan, la phrase graphique, qui est comprise entre une majuscule et un signe de ponctuation forte et qui peut correspondre à une ou plusieurs phrases syntaxiques autonomes.

De ce grand nombre de «phrases» dans les grammaires de référence découle, dans les grammaires scolaires, un amalgame entre critères sémantiques, syntaxiques et graphiques lorsque vient le temps de définir une phrase, ainsi que des divergences terminologiques et conceptuelles sur ses caractéristiques syntaxiques. Par exemple, s'ils s'entendent tous sur le fait qu'une phrase est une suite de mots ordonnée qui a du sens (critère sémantique), qui commence par une majuscule et se termine par un point (critère graphique), les auteurs de quelques grammaires du $3^{\mathrm{e}}$ cycle du primaire divergent quant à la description de ce qui constitue la phrase (qui est en fait la phrase syntaxique, sans que cela soit spécifié) : Lévesque, Bourbeau et Gosselin (2004) parlent de deux groupes obligatoires, le groupe sujet (GS) et le groupe du verbe (GV), et d'un groupe dont la présence n'est pas obligatoire, le groupe complément de phrase (GCP); Laporte et Rochon (2004) évoquent deux constituants obligatoires, le groupe sujet et le groupe du verbe, et parfois un troisième constituant, le groupe complément de phrase; Chartrand et Simard (2000) parlent aussi de deux constituants obligatoires, mais les nomment sujet et prédicat, et d'un constituant facultatif, le complément de phrase.

On peut dès lors se demander, d'une part, comment les enseignants du primaire conçoivent et présentent à leurs élèves cet objet complexe qu'est la notion de phrase et, d'autre part, quelle est la conception ${ }^{1}$ de la

1. À l'instar de Clément (1994) et de Giordan et De Vecchi (1987), nous retenons le terme de conceptions au sens de «structures mentales mises en œuvre face à des situations-problèmes particulières ». Entendu comme un quasi-synonyme de représentations, le terme de conceptions apparait en effet plus approprié quand l'objet de la représentation est réellement un concept, comme c'est le cas de la phrase (Anctil, 2010). Il comprend à la fois ce qui est juste et ce qui est erroné. 
phrase qui en résulte chez ces élèves. Or, très peu de recherches ont été menées à ce sujet, du moins en français. Nous en citerons trois.

En contexte québécois, Tremblay (2007) a observé, à travers des entrevues menées auprès d'enseignants du $3^{\mathrm{e}}$ cycle du primaire au sujet de la phrase de base et de ses constituants, que cette notion était souvent mal comprise. Les données recueillies dans son étude montrent que plusieurs des répondants interrogés confondent le verbe et le groupe verbal, ne connaissent pas la fonction de prédicat, ont du mal à différencier les compléments du verbe des compléments de phrase et utilisent encore le terme de «complément circonstanciel» pour décrire un des constituants de la phrase.

Du côté des élèves, mais en France, cette fois, Darras et Cauterman (1997) ont montré que les élèves de $6^{\mathrm{e}}$ année disposent de peu de critères pour justifier si les énoncés qu'on leur propose constituent des phrases. Leurs critères sont essentiellement sémantiques, parfois stylistiques ou prosodiques : pour eux, une phrase devrait vouloir dire quelque chose, être un énoncé complet, ne pas «avoir trop» (de quelque chose), dire quelque chose (par opposition à poser une question ou à donner un ordre). Qui plus est, à partir des mêmes énoncés, Boutet (1986) avait observé que les jugements d'acceptabilité que portent les élèves de 6 à 11 ans ne sont pas différents selon l'âge des sujets - autrement dit, que la conscience syntaxique n'évoluerait pas durant la scolarité primaire. On peut se demander si ce constat, pour le moins préoccupant, demeure actuel.

Pour documenter la conception de la phrase qu'ont les élèves du primaire et leurs enseignants, en contexte québécois, ainsi que le lien entre la conception des uns et celle des autres, nous avons mené, sur une période de deux années scolaires, une recherche collaborative ${ }^{2}$ (Desgagné, 1997) portant sur l'enseignement et l'apprentissage de la notion de phrase à l'aide de la littérature de jeunesse.

Cet article se donne pour objectifs :

1) de décrire quantitativement la performance relative à la conception de la phrase d'enseignants et d'élèves de la $1^{\text {re }}$ à la $6^{\mathrm{e}}$ année du primaire, ainsi que le lien entre la performance des premiers et des seconds;

2. Cette recherche a été financée par le Fonds québécois de recherche sur la société et la culture (projet $\mathrm{n}^{\circ}$ 144254), en collaboration avec le ministère de l'Éducation, du Loisir et du Sport du Québec, à qui les auteurs expriment leur reconnaissance. 
2) de décrire les types d'arguments dominants employés par les uns et les autres pour justifier leurs jugements de grammaticalité;

3) de documenter par l'étude du cas d'une classe le lien entre les arguments utilisés par l'enseignant et ceux évoqués par les élèves afin de nuancer qualitativement la relation entre les conceptions de l'un et des autres en matière de phrase.

\section{Méthodologie}

La recherche a été menée pendant les années scolaires 2011-2012 et 2012-2013 dans quatre écoles de la rive sud de Montréal auprès de 19 enseignants de tous les niveaux du primaire (15 en 2011-2012, 14 en 2012-2013, dont 10 suivis les deux années, à raison de cinq par cycle) et de leurs 494 élèves (273 en 2011-2012, 265 en 2012-2013 et 44 les deux années). Pendant la durée du projet, les enseignants ont participé à sept jours de formation par année, offerts par les chercheurs et deux conseillers pédagogiques de français de la commission scolaire. La formation portait notamment sur les concepts grammaticaux autour de la notion de phrase, mais également sur des dispositifs d'enseignement de l'écriture et sur des œuvres de littérature de jeunesse propices à l'observation de phénomènes syntaxiques.

Nous avons réalisé auprès des enseignants, à trois reprises pendant chaque année scolaire, une entrevue comportant deux tâches : l'une où ils devaient déterminer si les énoncés proposés étaient des phrases et justifier leur réponse (jugement de grammaticalité); l'autre où ils devaient repérer l'erreur syntaxique dans des phrases d'élèves en expliquant la nature de l'erreur (correction). Les tableaux 1 et 2 présentent les énoncés de chacune des deux tâches.

La première de ces tâches a également été demandée aux élèves au début et à la fin de l'année scolaire. Pour les enseignants, qui ont été rencontrés au moins trois fois (six dans le cas de ceux qui ont participé aux deux années du projet), des énoncés différents mais construits sur le même modèle ont été présentés lors de chaque passation, afin d'éviter que les enseignants mémorisent les items de l'entrevue d'une passation à l'autre.

Pour constituer un score à chaque tâche, les points ont été attribués de la façon suivante. Pour la tâche de jugement, un point était accordé lorsque le participant donnait la réponse attendue pour le jugement de grammaticalité (maximum de 15). Puis, les arguments utilisés pour justifier la réponse à cinq énoncés (les numéros $2,4,5,6$ et 8) se sont 
vu attribuer des points en fonction de leur pertinence; ces énoncés ont été choisis parce qu'ils représentaient des cas de figure variés : deux P autonomes grammaticales coordonnées, une $\mathrm{P}$ agrammaticale dont le prédicat est incomplet, une $\mathrm{P}$ grammaticale comportant un sujet et un prédicat très courts, une $\mathrm{P}$ grammaticale mais asémantique et une $\mathrm{P}$ grammaticale sans caractéristiques particulières. Les arguments évoquant les constituants obligatoires d'une $\mathrm{P}$ recevaient 3 points ; ceux de nature syntaxique mais ne faisant pas référence aux constituants recevaient 2 points; les arguments pertinents mais non syntaxiques (par exemple, ceux portant sur le sens) recevaient 1 point; les arguments vrais mais non pertinents (par exemple, ceux faisant référence à la classe d'un mot) recevaient 0 point; et les arguments faux se voyaient attribuer -1 . Les participants pouvant invoquer un nombre variable d'arguments, il n'y avait ni maximum ni minimum pour le pointage qui leur était attribué. Pour la tâche de correction, un point était accordé quand le participant repérait l'erreur (maximum de 7) ; un autre quand il proposait une correction pertinente (maximum de 7); et les arguments utilisés pour expliquer l'erreur et sa correction se voyaient attribuer 3, 2, 1, 0 ou -1 points comme dans la première tâche. Un score global numérique a été constitué pour chaque enseignant à chaque passation en additionnant les points obtenus dans les deux tâches; de la même façon, un score a également été calculé pour les élèves à partir des points obtenus à la tâche de jugement.

Parallèlement à cela, afin de pouvoir décrire la nature des justifications utilisées, pour les 15 énoncés de la tâche de jugement de grammaticalité, tous les arguments ont été codés selon qu'ils étaient syntaxiques, sémantiques ou autres (pragmatiques, esthétiques, etc.). De plus, pour chaque énoncé, un certain nombre d'arguments ont été identifiés comme «privilégiés » ou «contextuellement pertinents»; souvent, mais non exclusivement, de nature syntaxique, c'étaient les arguments que nous estimions les plus déterminants pour juger de la grammaticalité de chaque énoncé. Des exemples de ces justifications seront présentés dans la section «résultats».

Enfin, pour documenter les pratiques d'enseignement de la phrase adoptées par les enseignants, ces derniers ont été observés en classe à deux reprises chaque année de leur participation au projet. À ces occasions, les observations ont été consignées sous forme de notes mais surtout de photographies, qui reflètent les interventions les plus significatives de l'enseignant, les tâches effectuées par les élèves, le matériel utilisé, le climat général de la classe, etc. 


\begin{tabular}{|l|c|}
\hline \multicolumn{1}{|c|}{ Énoncé } & Réponse attendue \\
\hline 1. Aimes-tu le sport? & Oui \\
\hline 2. Camille prit son petit frère dans ses bras et elle l'embrassa. & Oui \\
\hline 3. J'aime jouer dehors. & Oui \\
\hline 4. Justin et Léa vont. & Non \\
\hline 5. L'eau coule. & Oui \\
\hline 6. La girafe est une fleur sucrée. & Oui \\
\hline 7. La délicieuse tarte aux fraises. & Non \\
\hline 8. Les enfants mangent de la soupe. & Oui \\
\hline 9. Mettre le gâteau au four 30 minutes. & Oui \\
\hline 10. Olivier espère que ses amis viendront à la fête. & Oui \\
\hline 11. Parce que je le sais. & Non \\
\hline 12. Quelle bonne idée! & Oui \\
\hline 13. Range ta chambre tout de suite. & Oui \\
\hline 14. Ses gants a perdu Maman. & Non \\
\hline $\begin{array}{l}\text { 15. Tous les dimanches matin, du début avril à la fin octobre, } \\
\text { ils se rendaient dans leur voiture à leur chalet au creux de la } \\
\text { montagne. }\end{array}$ & Oui \\
\hline
\end{tabular}

Tableau 1. - Énoncés proposés et réponses attendues dans la première tâche (jugement de grammaticalité).

\begin{tabular}{|l|l|}
\hline \multicolumn{1}{|c|}{ Énoncé proposé } & \multicolumn{1}{c|}{ Correction attendue } \\
\hline 1. La mère de Marie-Lise elle se dépêcha. & $\begin{array}{l}\text { La mère de Marie-Lise se dépêcha. } \\
\text { La mère de Marie-Lise, elle se dépêcha. } \\
\text { La mère de Marie-Lise, elle, se dépêcha. }\end{array}$ \\
\hline $\begin{array}{l}\text { 2. Quand il eut terminé, un tigre avec des } \\
\text { dents pointues rouges. }\end{array}$ & $\begin{array}{l}\text { Quand il eut terminé, un tigre avec des } \\
\text { dents pointues rouges est apparu (p. ex.). }\end{array}$ \\
\hline $\begin{array}{l}\text { 3. Sa mère était désespérée plus d'espoir } \\
\text { presque plus d'argent. }\end{array}$ & $\begin{array}{l}\text { Sa mère était désespérée, elle n'avait plus } \\
\text { d'espoir, presque plus d'argent. (p. ex.). }\end{array}$ \\
\hline 4. Marie cueille. & Marie cueille des fruits (p. ex.) \\
\hline $\begin{array}{l}\text { 5. J'allai chercher ma mère pour lui dire } \\
\text { en courant que la chatte était en train } \\
\text { d'accoucher. }\end{array}$ & $\begin{array}{l}\text { J'allai chercher ma mère en courant } \\
\text { pour lui dire que la chatte était en train } \\
\text { d'accoucher. }\end{array}$ \\
\hline $\begin{array}{l}\text { 6. Il alla répondre à porte en robe de } \\
\text { chambre. }\end{array}$ & $\begin{array}{l}\text { Il alla répondre à la porte en robe de } \\
\text { chambre. }\end{array}$ \\
\hline 7. Mais elle a pas écouté ses parents. & Mais elle n'a pas écouté ses parents. \\
\hline
\end{tabular}

Tableau 2. - Énoncés proposés et corrections attendues dans la deuxième tâche (correction). 


\section{Résultats}

\subsection{La performance des enseignants et des élèves quant à la conception de la phrase}

Nous commencerons par examiner les résultats des participants à la recherche en termes quantitatifs. Le tableau 3 présente les scores moyens des enseignants aux 6 moments de passation, pour chaque aspect des deux tâches et au total. Les trois mesures prises chez les enseignants qui se sont joints au projet la deuxième année ont été ajoutées aux temps 1 , 2 et 3 des enseignants présents dès la première année, puisque dans tous les cas il s'agissait de leurs trois premières passations.

\begin{tabular}{|l|c|c|c|c|c|c|}
\hline & Temps 1 & Temps 2 & Temps 3 & Temps 4 & Temps 5 & Temps 6 \\
\hline Tâche 1, jugement & 13,06 & 13,89 & 14,06 & 14,00 & 13,70 & 14,30 \\
\hline Tâche 1, justification & 12,25 & 13,95 & 14,67 & 14,92 & 15,60 & 14,90 \\
\hline Tâche 2, repérage de 1'erreur & 5,69 & 6,11 & 6,78 & 6,33 & 6,40 & 6,90 \\
\hline Tâche 2, correction & 3,41 & 4,45 & 4,86 & 3,75 & 4,95 & 4,85 \\
\hline Tâche 2, justification & 5,88 & 6,37 & 5,50 & 4,83 & 7,20 & 6,80 \\
\hline Total & 40,28 & 44,76 & 45,86 & 43,83 & 47,85 & 47,75 \\
\hline
\end{tabular}

Tableau 3. - Scores moyens des enseignants aux 6 moments de passation (temps 1 à 6).

Une analyse de la variance à mesures répétées estimée à l'aide d'un modèle linéaire mixte a permis de comparer les différents scores d'un moment de passation à l'autre. Pour le score global, la différence observée entre les scores des enseignants aux différents moments de passation est significative la première année de leur participation au projet $(F(2,28)=12,68, p=0,0001)$. De la même façon, la différence entre les scores aux six moments de passation est significative $(F(5,40)=3,45$, $p=0,0111)$; plus précisément, les scores au temps 5 et au temps 6 sont significativement supérieurs au score au temps 1 . Les différences observées pour les aspects des deux tâches aux différents moments de passation sont également significatives, sauf pour la justification, aussi bien pour la première année de participation au projet (tâche 1 , jugement : $F(2,28)=10,17, p=0,0005$; tâche 2, repérage : $F(2,28)=9,61, p=0,0007$; tâche 2, correction : $F(2,28)=9,37, p=0,0008)$ que pour les six moments de passation (tâche 1, jugement : $F(5,40)=2,78, p=0,0300$; tâche 2, repérage : $F(5,40)=6,54, p=0,0002$; tâche 2, correction : $F(5,40)=5,82$, $p=0,0004)$. Cela signifie que la formation a significativement contribué à une amélioration de la conception de la phrase chez les enseignants et 
que ce progrès est particulièrement flagrant chez ceux qui ont participé aux deux années du projet.

Le tableau 4 présente les scores moyens obtenus par les élèves aux deux moments de passation à chaque niveau scolaire, pour chaque aspect de la tâche de jugement et au total.

\begin{tabular}{|l|c|c|c|c|c|c|c|c|c|c|c|c|}
\hline & \multicolumn{2}{|c|}{$1^{\text {re }}$} & \multicolumn{2}{c|}{$2^{\mathrm{e}}$} & \multicolumn{2}{c|}{$3^{\mathrm{e}}$} & \multicolumn{2}{c|}{$4^{\mathrm{e}}$} & \multicolumn{2}{|c|}{$5^{\mathrm{e}}$} \\
$\left(\mathrm{N}=56,58^{*}\right)$ & $(\mathrm{N}=89,88)$ & \multicolumn{3}{c|}{$6^{\mathrm{e}}$} \\
$(\mathrm{N}=47,47)$ & $(\mathrm{N}=122,125)$ & $(\mathrm{N}=38,38)$ & $(\mathrm{N}=126,109)$ \\
\hline & $\mathrm{T} 1$ & $\mathrm{~T} 2$ & $\mathrm{~T} 1$ & $\mathrm{~T} 2$ & $\mathrm{~T} 1$ & $\mathrm{~T} 2$ & $\mathrm{~T} 1$ & $\mathrm{~T} 2$ & $\mathrm{~T} 1$ & $\mathrm{~T} 2$ & $\mathrm{~T} 1$ & $\mathrm{~T} 2$ \\
\hline Jugement & 10,13 & 9,78 & 9,84 & 10,63 & 10,72 & 10,91 & 10,82 & 11,49 & 10,71 & 11,92 & 11,19 & 11,56 \\
\hline Justification & 0,07 & 1,43 & 1,26 & 2,53 & 2,66 & 4,91 & 3,41 & 5,65 & 3,82 & 7,39 & 6,10 & 9,05 \\
\hline Total & 10,20 & 11,21 & 11,10 & 13,16 & 13,38 & 15,83 & 14,23 & 17,14 & 14,53 & 19,32 & 17,29 & 20,61 \\
\hline
\end{tabular}

* Le premier nombre correspond au $\mathrm{N}$ au $\mathrm{T} 1$, le second au $\mathrm{T} 2$, le nombre d'élèves par niveau ayant varié en cours d'année.

Tableau 4. - Scores moyens des élèves de chaque niveau scolaire aux 2 moments de passation ( $\left.T_{1}, T_{2}\right)$.

L'analyse de variance a révélé une interaction significative entre le temps de passation et le niveau scolaire, aussi bien pour le score global que pour chaque aspect de la tâche (jugement : $F(5,447)=3,13$, $p=0,0086$; justification : $F(5,447)=2,51, p=0,0297$; total $: F(5,447)=$ $2,69, p=0,0206)$. La différence est significative entre les deux moments de passation en $2^{\mathrm{e}}$, en $4^{\mathrm{e}}$ et en $5^{\mathrm{e}}$ année pour le jugement; à tous les niveaux pour la justification; à tous les niveaux sauf en $1^{\text {re }}$ année pour le score global. Ces résultats montrent que la capacité des élèves du primaire à porter un jugement de grammaticalité sur des énoncés et à le justifier s'améliore significativement entre le début et la fin d'une année scolaire (dans une moindre mesure en $1^{\text {re }}$ année), ainsi que d'un niveau scolaire à l'autre.

Nous avons voulu savoir si la performance des enseignants aux tâches sur la phrase que nous leur avons proposées dans l'entrevue était reliée au progrès de leurs élèves. Pour ce faire, nous avons subdivisé les enseignants en trois catégories (forts, moyens, faibles) selon leur résultat à l'entrevue sur la phrase au temps 1 pour ensuite étudier au moyen d'un modèle mixte la relation entre leur performance en début d'année et le progrès de leurs élèves. Il appert qu'aucune différence significative n'a été détectée. En d'autres termes, les enseignants qui ont la conception de la phrase la plus conforme aux savoirs de référence en début d'année scolaire n'amènent pas davantage que les autres leurs élèves à progresser dans leur conception de la phrase. Il est impossible 
d'établir un lien quantitatif entre les scores relatifs à la conception de la phrase des enseignants et ceux de leurs élèves.

\subsection{Les arguments utilisés pour justifier le jugement}

Penchons-nous maintenant plus spécifiquement sur les arguments utilisés par les enseignants et les élèves pour justifier leurs jugements de grammaticalité. Ces arguments ont été classés en trois catégories. Les arguments syntaxiques incluent ceux qui font référence au nombre de mots ou à la longueur de l'énoncé; au fait qu'il manque quelque chose (ou qu'il ne manque rien) dans l'énoncé; à la présence ou à l'absence d'un verbe, d'un GV, d'un nom, d'un GN, d'un sujet, d'un prédicat, d'un complément de phrase ou d'autres classes de mots que le nom ou le verbe; à la complétude syntaxique de la phrase; au fait que l'énoncé corresponde à plus d'une phrase; au type ou à la forme de la phrase; à la ponctuation; à la structure de la phrase. Les arguments sémantiques sont ceux qui font allusion au fait que l'énoncé ait du sens ou beaucoup de sens, qu'il n'en ait pas ou qu'il en manque. Les «autres» arguments sont ceux à caractère pragmatique (la référence à une situation de communication déjà vécue par l'élève ou la référence à l'intégration de cet énoncé dans l'usage), prosodique (la référence aux pauses ou à l'intonation) ou esthétique («ça sonne bien», par exemple), ceux liés à la validité référentielle (c'est-à-dire aux connaissances de l'élève sur le monde), ainsi que tout autre argument non prévu dans ce qui précède. Le tableau 5 présente le pourcentage des arguments de chacun de ces trois types aux différents moments de passation, pour les enseignants et les élèves (cette fois, les résultats des enseignants ont été distingués selon qu'ils ont été recueillis la $1^{\text {re }}$ ou la $2^{\mathrm{e}}$ année de la recherche).

\begin{tabular}{|l|c|c|c|c|c|c|c|c|c|}
\hline & \multicolumn{3}{|c|}{ T1 } & \multicolumn{3}{c|}{ T2 } & \multicolumn{3}{c|}{ T3 } \\
\hline & Synt. & Sém. & Autres & Synt. & Sém. & Autres & Synt. & Sém. & Autres \\
\hline Enseignants an 1 & $63 \%$ & $21 \%$ & $16 \%$ & $71 \%$ & $18 \%$ & $11 \%$ & $71 \%$ & $12 \%$ & $16 \%$ \\
\hline Enseignants an 2 & $75 \%$ & $18 \%$ & $7 \%$ & $78 \%$ & $13 \%$ & $8 \%$ & $74 \%$ & $14 \%$ & $12 \%$ \\
\hline Élèves $1^{\text {re }}$ année & $34 \%$ & $25 \%$ & $41 \%$ & & & & $53 \%$ & $25 \%$ & $22 \%$ \\
\hline Élèves $2^{\mathrm{e}}$ année & $53 \%$ & $22 \%$ & $25 \%$ & & & & $61 \%$ & $23 \%$ & $16 \%$ \\
\hline Élèves $3^{\mathrm{e}}$ année & $61 \%$ & $22 \%$ & $17 \%$ & & & & $71 \%$ & $19 \%$ & $9 \%$ \\
\hline Élèves $4^{\mathrm{e}}$ année & $69 \%$ & $20 \%$ & $11 \%$ & & & & $72 \%$ & $18 \%$ & $10 \%$ \\
\hline Élèves $5^{\mathrm{e}}$ année & $77 \%$ & $11 \%$ & $12 \%$ & & & & $71 \%$ & $17 \%$ & $12 \%$ \\
\hline Élèves $6^{\mathrm{e}}$ année & $77 \%$ & $14 \%$ & $9 \%$ & & & & $76 \%$ & $15 \%$ & $9 \%$ \\
\hline
\end{tabular}

Tableau 5. - Pourcentage des arguments de chaque type aux 3 moments de passation chez les enseignants et les élèves. 
Bien que le cadre d'analyse de la grammaire actuelle privilégie les arguments syntaxiques pour pouvoir juger de la grammaticalité d'un énoncé, force est de constater que tous les arguments catégorisés comme syntaxiques ne sont pas également pertinents; par exemple, la référence à une classe de mots comme l'adjectif ou l'adverbe n'est aucunement utile pour déterminer si un énoncé est une phrase ou non. Nous avons donc considéré, à l'intérieur des arguments syntaxiques, certains arguments comme «privilégiés» parce qu'ils étaient plus robustes pour juger de la grammaticalité d'un énoncé : ce sont ceux qui faisaient référence à la présence des constituants obligatoires d'une phrase (exprimés en termes de constituants, de sujet, de prédicat, de GV ou de GN). Nous avons considéré d'autres arguments comme «contextuellement pertinents » parce qu'ils s'avéraient utiles vu les caractéristiques de l'énoncé soumis (par exemple, la référence au type de phrase pour l'énoncé interrogatif Aimes-tu le sport? ou le fait que le sens soit incomplet dans l'énoncé Justin et Léa vont). Le tableau 6 présente le pourcentage des arguments privilégiés et contextuellement pertinents aux différents moments de passation, pour les enseignants et les élèves.

\begin{tabular}{|l|c|c|c|}
\hline & $\mathrm{T} 1$ & $\mathrm{~T} 2$ & $\mathrm{~T} 3$ \\
\hline Enseignants an 1 & $33 \%$ & $35 \%$ & $41 \%$ \\
\hline Enseignants an 2 & $39 \%$ & $44 \%$ & $38 \%$ \\
\hline Élèves $1^{\text {re }}$ année & $4 \%$ & & $13 \%$ \\
\hline Élèves $2^{\mathrm{e}}$ année & $10 \%$ & & $16 \%$ \\
\hline Élèves $3^{\mathrm{e}}$ année & $13 \%$ & & $18 \%$ \\
\hline Élèves $4^{\mathrm{e}}$ année & $16 \%$ & & $24 \%$ \\
\hline Élèves $5^{\mathrm{e}}$ année & $24 \%$ & & $31 \%$ \\
\hline Élèves $6^{\mathrm{e}}$ année & $24 \%$ & & $30 \%$ \\
\hline
\end{tabular}

Tableau 6. - Pourcentage des arguments privilégiés et contextuellement pertinents aux 3 moments de passation chez les enseignants et les élèves.

De façon générale, on constate que les élèves utilisent une plus forte proportion d'arguments syntaxiques à la fin de l'année qu'au début, sauf en $5^{\mathrm{e}}$ et en $6^{\mathrm{e}}$ années. De plus, le pourcentage d'arguments syntaxiques augmente d'un niveau scolaire à l'autre. En $5^{\mathrm{e}}$ et en $6^{\mathrm{e}}$ années, ce pourcentage chez les élèves est sensiblement équivalent à celui des enseignants. La même tendance s'observe pour les arguments privilégiés et contextuellement pertinents. Cependant, les enseignants utilisent une plus forte proportion de ces arguments que les élèves. 


\subsection{Les liens entre les arguments de l'enseignant et ceux des élèves : étude d'un cas}

On a déjà constaté qu'il n'y a pas de lien quantitatif entre le résultat obtenu par les enseignants en début d'année et le progrès de leurs élèves dans les tâches qui leur ont été proposées sur la phrase. Mais qu'en est-il d'éventuels liens qualitatifs? En d'autres termes, y a-t-il un lien entre la nature des arguments invoqués par un enseignant et ceux employés par ses élèves? Quelles relations existe-t-il entre les pratiques d'enseignement de la phrase adoptées par un enseignant et les progrès des élèves?

Pour répondre à ces questions, nous allons étudier le cas d'un enseignant de $1^{\text {re }}$ année du $1^{\text {er }}$ cycle, Yvan Guy, et des 18 élèves qui étaient dans sa classe la $2^{\mathrm{e}}$ année du projet. Yvan Guy a été choisi pour deux principales raisons. D'abord, sa performance dans l'entrevue sur la phrase était non seulement supérieure à la moyenne du groupe d'enseignants de la $2^{\mathrm{e}}$ année du projet, mais elle est restée constante aux trois moments de passation : chaque fois, $81 \%$ ou $82 \%$ de ses arguments étaient syntaxiques; et la proportion de ses arguments considérés comme privilégiés ou contextuellement pertinents était de 55\%, 45\% et 54\%. Ensuite, l'enseignement d'Yvan Guy se distinguait, selon nous, par sa qualité et sa densité de contenus syntaxiques; en effet, Yvan Guy a largement dépassé les prescriptions du programme pour son niveau scolaire, utilisant, par exemple, le concept de prédicat, qui est prévu en $4^{\mathrm{e}}$ année du primaire (MELS, 2009).

Le tableau 7 présente le type d'arguments utilisés par les élèves d'Yvan Guy au début et à la fin de l'année dans l'entrevue sur la phrase.

\begin{tabular}{|l|c|c|}
\cline { 2 - 3 } \multicolumn{1}{c|}{} & T1 & T3 \\
\hline Arguments syntaxiques & $23 \%$ & $49 \%$ \\
\hline Arguments sémantiques & $33 \%$ & $24 \%$ \\
\hline Autres arguments & $43 \%$ & $27 \%$ \\
\hline Arguments privilégiés et contextuellement pertinents & $5 \%$ & $11 \%$ \\
\hline
\end{tabular}

Tableau 7. - Pourcentage des différents types d'arguments utilisés par les élèves de la classe d'Yvan Guy aux deux temps de passation.

Les élèves de la classe d'Yvan Guy ont progressé dans l'utilisation d'arguments syntaxiques (passant de 23 à $49 \%$ ), ainsi que dans leur utilisation d'arguments privilégiés et contextuellement pertinents (en hausse de 5 à $11 \%$ ). Rappelons qu'il s'agit d'élèves de $1^{\text {re }}$ année du primaire qui, selon les prescriptions ministérielles, seraient censés étudier 
pour seuls concepts syntaxiques quelques caractéristiques d'une phrase (l'expression d'un sens, la présence de tous les mots, l'ordre des mots, la présence d'un verbe conjugué la plupart du temps), le fait qu'elle commence par une majuscule et se termine par un point et la négation ne ...pas (MELS, 2009).

Les pratiques observées dans la classe d'Yvan Guy permettent de mieux comprendre ce qui a aidé ses élèves à avoir recours à des arguments syntaxiques si fréquemment. La figure suivante illustre une pratique observée dans la classe d'Yvan Guy en novembre 2011.

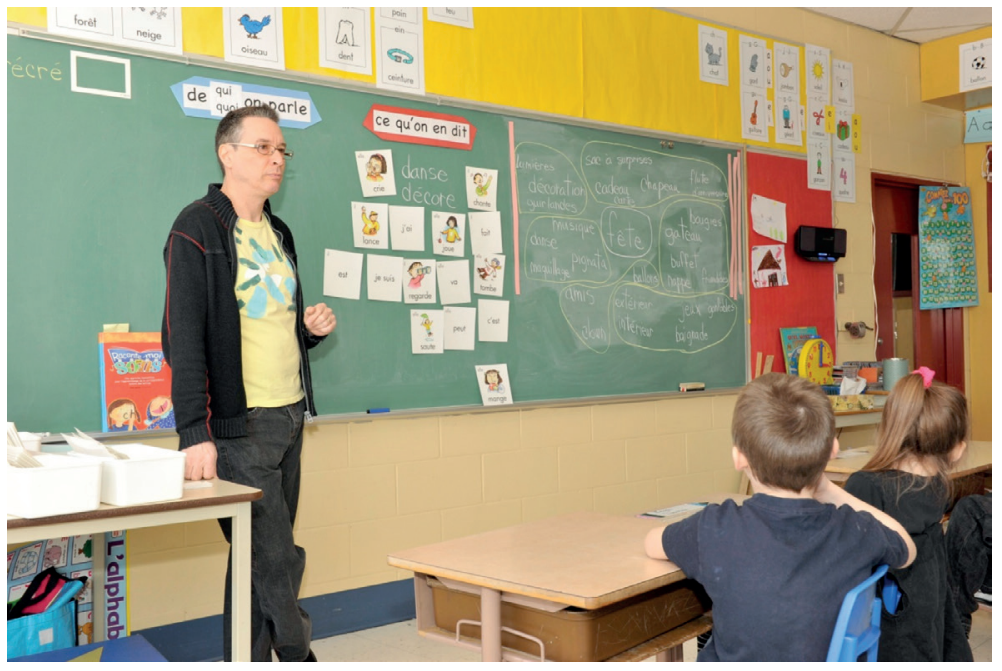

Fig. 1 - Planification de l'écriture d'un texte dans la classe d'Yvan Guy.

Au total pendant le projet, quatre observations ont été réalisées dans sa classe. Lors de trois d'entre d'elles, les élèves ont écrit en se servant de ce qu'ils avaient observé dans des œuvres lues par Yvan Guy. Le recours aux textes mentors (Anderson, 2006; Culham, 2014; Dorfman \& Cappelli, 2007; Fletcher, 2011; Gallagher, 2011) a été fortement encouragé dans le cadre de notre recherche et Yvan Guy a adopté rapidement ce type de pratique, qui consiste à prendre appui sur l'expertise d'un auteur en observant sa manière d'écrire, notamment en termes syntaxiques, pour générer un nouveau texte. C'est seulement lors de la première observation qu'il a fait écrire les élèves sans le recours aux textes mentors. Toutefois, les élèves avaient accès à un étayage de grande qualité de la part de leur enseignant.

En effet, lorsqu'Yvan Guy fait écrire ses élèves, il les aide à planifier leurs idées en les organisant à l'aide de deux cartons. Sur le premier, 
il est écrit de qui (ou de) quoi on parle; sur le second, ce qu'on en dit, ce qui permet de désigner sémantiquement les deux constituants obligatoires de la phrase. Par exemple, lors de la planification de l'écriture d'un texte à propos d'une fête, il a d'abord fait une tempête d'idées pour déterminer de quoi les élèves allaient pouvoir parler. C'est ce que l'on trouve à droite sur la figure 1. Puis, pour déterminer ce que l'on en dit, il a utilisé la boite dans laquelle il range les verbes et, avec les élèves, il a choisi de mettre au tableau sous le carton ce qu'on en dit ceux dont le sens pouvait convenir dans le cadre de ce texte.

Dans son enseignement lexical, Yvan Guy permet à ses élèves d'organiser leurs connaissances à l'aide des classes de mots en les invitant à classer dans les boites prévues à cet effet les verbes, les noms, les adjectifs, les déterminants et les mots invariables, qui regroupent toutes les autres classes de mots. Ce travail lexical lui permet d'établir des liens entre le sens et la syntaxe. Par exemple, implicitement, les élèves sont amenés à déduire que ce qu'on en dit implique un verbe et correspond au prédicat, terme qu'Yvan Guy introduit d'ailleurs un peu plus tard dans l'année.

\section{Discussion et conclusion}

Notre recherche collaborative avait notamment pour objectif de donner à des enseignants une formation sur un objet grammatical central, la notion de phrase, afin qu'ils en aient une meilleure connaissance et qu'ils développent de meilleures pratiques pour l'enseigner à leurs élèves. Les résultats montrent que les connaissances des enseignants sur la phrase se sont significativement améliorées à la suite de la formation. Cela permet donc de conclure à l'importance de la formation continue chez les enseignants en matière de didactique de la grammaire. Cependant, il faut souligner que les enseignants qui ont participé au projet le faisaient sur une base volontaire et se sont révélés particulièrement engagés, ce qui a grandement contribué au succès de la formation. D'aucuns pourraient se questionner sur l'opportunité de consacrer autant de ressources à un seul objet, en l'occurrence la notion de phrase. Sans nier le cout d'un tel investissement, nous soulignerons que la notion de phrase est centrale dans la description de la langue et qu'elle est propice à la mobilisation de nombreux autres concepts grammaticaux; il est donc raisonnable d'espérer que ce travail sur la phrase aura chez les enseignants des effets positifs sur d'autres notions grammaticales et que l'investissement en valait la peine. 
Bien que nous n'ayons pu établir de lien quantitatif entre la maitrise conceptuelle des enseignants et les progrès de leurs élèves, nous constatons tout de même chez ces derniers, entre le début et la fin de l'année ainsi que d'un niveau scolaire à l'autre, une proportion croissante d'arguments syntaxiques et, surtout, d'arguments privilégiés et contextuellement pertinents. Au $3^{\mathrm{e}}$ cycle $\left(5^{\mathrm{e}}\right.$ et $6^{\mathrm{e}}$ années), les élèves ont recours à une proportion d'environ $30 \%$ de ce dernier type d'arguments, proportion qui se rapproche de celle d'environ $40 \%$ observée chez leurs enseignants, adultes et scripteurs experts. Nos résultats vont à l'encontre de ce que Darras et Cauterman (1997) ont observé, à savoir que les arguments des élèves sont essentiellement sémantiques, ainsi que des conclusions de Boutet (1986), qui affirme qu'il n'y a pas de différence entre les jugements d'acceptabilité à propos de phrases portés par des élèves de différents niveaux du primaire. Cela tient peut-être au fait que l'enseignement de la grammaire dispensé en contexte québécois depuis 2001 repose nettement plus sur la dimension syntaxique que ce n'était le cas à l'époque où ces études ont été réalisées.

Cette absence de lien quantitatif entre la performance des enseignants aux tâches sur la phrase en début d'année et le progrès de leurs élèves demeure troublante : elle pourrait suggérer que les connaissances disciplinaires de l'enseignant n'affectent pas les apprentissages des élèves. Au contraire, nous faisons l'hypothèse que ces connaissances sont nécessaires, mais non suffisantes à un enseignement de qualité; des compétences didactiques sont également requises, compétences que nous avons cherché à développer durant la formation et que nous avons documentées de façon qualitative sans que nos épreuves puissent en rendre compte quantitativement. Par ailleurs, dans une perspective statistique, un plus grand nombre d'enseignants aurait peut-être permis l'obtention de résultats significatifs.

Enfin, l'accent mis sur la syntaxe dans l'enseignement ne peut ni ne doit faire abstraction du sens. Comme on l'a constaté dans la classe d'Yvan Guy, c'est en entrant par la dimension sémantique qu'il a offert à ses élèves une première prise sur les concepts syntaxiques de sujet et de prédicat, ce qui leur a permis de poser les assises de ces concepts centraux pourtant à l'étude trois ans plus tard selon les prescriptions ministérielles québécoises. Cela pourrait inviter à reconsidérer la répartition des concepts à l'étude au fil de la scolarisation primaire en s'appuyant sur des données empiriques, répartition qui demeurera inévitablement artificielle vu les interrelations nombreuses entre les différents concepts grammaticaux et dimensions de la langue. 
L'intuition d'Yvan Guy, qui utilise une entrée sémantique pour approcher la syntaxe, nous apparait féconde, puisqu'elle permet de travailler efficacement la syntaxe dès le début du premier cycle. Bien entendu, plus les élèves progresseront dans leur scolarité primaire puis secondaire, plus l'enseignement grammatical reposera sur des concepts syntaxiques, en accord avec les préceptes de la grammaire moderne. Toutefois, cet enseignement ne devrait pas, selon nous, évacuer la dimension sémantique, car, rappelons-le, la langue demeure au service de l'énonciation.

\section{RÉFÉRENCES BIBLIOGRAPHIQUES}

ANCTIL, Dominic. (2010). L'erreur lexicale au secondaire : analyse d'erreurs lexicales d'élèves de $3^{e}$ secondaire et description du rapport à l'erreur lexicale d'enseignants de français (Thèse de doctorat non publiée). Université de Montréal, Montréal.

Anderson, Jeff. (2006). Zooming in and Zooming Out: Putting Grammar in Context into Context. English Journal, 95(5), 28-34.

Boivin, Marie-Claude \& Pinsonneault, Reine. (2008). La grammaire moderne. Description et éléments pour sa didactique. Montréal, Canada : Beauchemin/Chenelière Éducation.

Boutet, Josiane. (1986). Jugements d'acceptabilité et raisonnements métalinguistiques. Revue ELA, 62, 40-58.

Chartrand, Suzanne-Geneviève \& Simard, Claude. (2000). Grammaire de base. Montréal, Canada : ERPI.

Clément, Pierre. (1994). Représentations, conceptions, connaissances. Dans A. Giordan, Y. Girault \& P. Clément (dir.), Conceptions et connaissances (p. 15-45). Berne, Suisse : Peter Lang.

Culnam, Ruth. (2014). The Writing Thief: Using Mentor Texts to Teach the Craft of Writing. Newark, DE : International Reading Association.

DarRas, Francine \& CAUTERMAn, Marie-Michèle. (1997). Mais qu'est-ce qu'une phrase? Recherches, 26, 209-219.

Desgagné, Serge. (1997). Le concept de recherche collaborative : l'idée d'un rapprochement entre chercheurs universitaires et praticiens enseignants. Revue des sciences de l'éducation, 23(2), 371-393.

Desnoyers, Annie. (2001). Les grammaires (fascicules 1 à 4). Montréal, Canada : CCDMD.

Dorfman, Lynne R. \& CAPpell, Rose. (2007). Mentor Texts: Teaching Writing Through Children's Literature, K-6. Portland, ME : Stenhouse Publishers.

Fletcher, Ralph. (2011). Mentor Author, Mentor Texts: Short Texts, Craft Notes, and Practical Classroom Uses. Portsmouth, NJ : Heinemann. 
Gallagher, Kelly. (2011). Write Like This: Teaching Real-World Writing Through Modeling \& Mentor Texts. Portland, ME : Stenhouse Pub.

Giordan, André \& De Vecchi, Gérard. (1987). Les origines du savoir. Des conceptions des apprenants aux concepts scientifiques. Neuchâtel, Suisse : Delachaux et Niestlé.

Groupe DIEPE. (1995). Savoir écrire au secondaire. Louvain, Belgique : De Boeck Université.

LAPORTE, Myriam \& Rochon, Ginette. (2004). Grammaire jeunesse, $3^{e}$ cycle du primaire. Montréal, Canada : CEC.

Lefrançois, Pascale, Laurier, Michel D., Lazure, Roger \& Claing, Robert. (2008). Évaluation de l'efficacité des mesures visant l'amélioration du français écrit du primaire à l'université. Montréal, Canada : Office québécois de la langue française (Suivi de la situation linguistique, étude 9).

Lévesque, Clément, Bourbeau, Geneviève \& Gosselin, Marie-Hélène. (2004). Grammaire française au primaire, le petit guide du $3^{e}$ cycle. Montréal, Canada : Grand Duc HRW.

Ministère de L'Éducation, du Loisir et du Sport. (2009). Progression des apprentissages au primaire en français, langue d'enseignement. Québec, Canada : Gouvernement du Québec.

Ministère de L'Éducation, Du Loisir ET Du Sport. (2012). Évaluation du Plan d'action pour l'amélioration du français. Québec, Canada : Gouvernement du Québec.

NADEAU, Marie \& FISHER, Carole. (2006). La grammaire nouvelle : la comprendre et l'enseigner. Montréal, Canada : Gaëtan Morin éditeur/ Chenelière Éducation.

Riegel, Martin, Pellat, Jean-Christophe \& Rioul, René. (2004). Grammaire méthodique du français ( $3^{\mathrm{e}}$ éd.). Paris : PUF.

Tremblay, Jean-François. (2007). Conceptions et pratiques déclarées relatives à l'enseignement des groupes constituants de la phrase de base au troisième cycle du primaire (Mémoire de maitrise non publié). Université de Montréal, Montréal. 